\title{
Da cantina à UAN: Transições no âmbito da alimentação escolar
}

\author{
From the cafeteria to the nutrition and food unit (UAN): The transitions in the context of school \\ feeding \\ De la cafetería a la unidad de nutrición y alimentación (UAN): Transiciones en el contexto de la \\ alimentación escolar
}

Recebido: 26/04/2021 | Revisado: 05/05/2021 | Aceito: 08/05/2021 | Publicado: 23/05/2021

\author{
Alessandra Santiago da Silva \\ ORCID: https://orcid.org/0000-0003-1364-9303 \\ Universidade Federal da Bahia, Brasil \\ E-mail: alessandrasantiago@ @otmail.com \\ Lígia Amparo da Silva Santos \\ ORCID: https://orcid.org/0000-0002-6925-6421 \\ Universidade Federal da Bahia, Brasil \\ E-mail: amparo@ufba.br \\ Lilian Miranda Magalhães \\ ORCID: https://orcid.org/0000-0003-4964-052X \\ Universidade Federal da Bahia, Brasil \\ E-mail: lilianmirandam@gmail.com \\ Maria da Purificação Nazaré Araújo \\ ORCID: https://orcid.org/0000-0002-8279-4769 \\ Universidade Federal da Bahia, Brasil \\ E-mail: puri@ufba.br
}

\begin{abstract}
Resumo
O presente estudo tem como objetivo analisar o processo de transição da alimentação escolar considerando as modificações na estrutura do seu espaço de produção. Foi realizada uma pesquisa de cunho etnográfico, no período de outubro a dezembro de 2014, em uma escola pública de bairro popular da cidade de Salvador (BA), tendo como principais interlocutoras as merendeiras da instituição. Para estabelecer um diálogo com os dados empíricos (observação participante e entrevistas) produzidos em campo, foram analisadas publicações científicas e documentos oficiais. Os resultados obtidos evidenciam que a transição de "cantina" para "Unidade de Alimentação e Nutrição" prevaleceu muito mais no campo conceitual do que no estrutural, o que gera dois espaços simbólicos: a "cozinha ideal", plenamente alinhada à legislação e conteúdo das capacitações, e a "cozinha real", em que são realizadas as práticas cotidianas. Avanços posteriores na política relacionada ao PNAE devem considerar a escuta de todos envolvidos no processo, além de uma reflexão crítica a respeito da sua efetividade no âmbito escolar.
\end{abstract}

Palavras-chave: Alimentação escolar; Merendeiras; PNAE; Segurança alimentar e nutricional.

\begin{abstract}
This study aims to analyze the school feeding transition process by taking into account the changes in the structure of its production space. An ethnographic research was carried out in a public school located in a low-income neighborhood in the city of Salvador (Bahia, Brazil) from October to December 2014 by mainly interviewing the school lunch handlers in that institution. In order to establish a dialogue with the empirical data gathered in the field, scientific publications and official documents were analyzed. Results show the transition from the "Cafeteria" to the "Nutrition and Food Unit" was shown to prevail much more at the conceptual than at the structural level, thus producing two symbolic spaces: the "ideal kitchen", fully in line with both the legislation and the content regarding the handlers' training, and the "real kitchen", in which everyday practices take place. Further advances in PNAErelated policies should consider listening to all personnel involved in the process and undertake a critical reflection about their effectiveness in school settings.

Keywords: School feeding; School food handlers; Brazilian school feeding program-PNAE; Food and nutrition security.

\section{Resumen}

El presente estudio tiene como objetivo analizar el proceso de transición de la alimentación escolar teniendo en cuenta los cambios en la estructura de su espacio de producción. Se realizó una investigación etnográfica, de octubre a diciembre de 2014, en una escuela pública de un barrio popular de la ciudad de Salvador (Bahia, Brasil), con los manipuladores de almuerzos escolares de la institución como principales interlocutores. Con el fin de entablar un
\end{abstract}


diálogo con los datos empíricos (observación participante y entrevistas) producidos en el campo, se analizaron publicaciones científicas y documentos oficiales. Los resultados obtenidos muestran que la transición de la "cafetería" a la "unidad de nutrición y alimentación" predominó mucho más en el ámbito conceptual que en el estructural, produciendo así dos espacios simbólicos: la "cocina ideal", totalmente alineada con el la legislación y los contenidos sobre la formación de los manipuladores, y la "cocina real", en la que se desarrollan las prácticas diarias. Los avances posteriores en la política relacionada con el PNAE deben considerar escuchar a todos los involucrados en el proceso, además de una reflexión crítica sobre su efectividad en el entorno escolar.

Palabras clave: Alimentación escolar; Manipuladores de alimentos escolares; Programa brasileño de alimentación escolar - PNAE; Seguridad alimentaria y nutricional.

\section{Introdução}

Até meados do século XX, no Brasil, a alimentação de estudantes na escola não possuía qualquer sistematização ou participação do governo. O local era destinado exclusivamente à realização de atividades de ensino. Movidos pela ideia de que a fome afetava a aprendizagem, por iniciativa própria, professores e pessoas da comunidade começaram a atuar de modo voluntário para fornecer alimentos adquiridos por meio de doações (Coimbra, Meira \& Starling, 1982; Fernandes, 2012; Gomes, 2014; Moysés \& Collares, 1997).

Nas últimas seis décadas, as ações pontuais e momentâneas foram ampliadas sob a forma de campanhas, que antecederam o Programa Nacional de Alimentação do Escolar (PNAE) (Fernandes, 2012). Desse modo, as ações nesse âmbito emergiram do cunho caritativo, passando pelo caráter assistencialista até chegar à atual condição de suplementar (Lei $\mathrm{n}$. 11.947, 2009). Atualmente, o aumento significativo do volume e complexidade dos alimentos preparados nas escolas (Coimbra, Meira \& Starling, 1982; Chaves, 2000; Costa, 2001; Fernandes, 2012) demandou a ampliação de ações no sentido de instrumentalizar o trabalho das merendeiras, para acompanhar o processo de expansão do Programa.

Nesse contexto, houve mudança na designação do espaço destinado à produção e distribuição da alimentação escolar: de "cantina" para "Unidade de Alimentação e Nutrição" (UAN), subsidiada por marcos legais e científicos (Cardoso, Souza \& Santos, 2005; Colombo, Oliveira \& Silva, 2009; Da Cunha, Stedefeldt \& De Rosso, 2012; Danelon, Danelon \& Silva, 2006; Lima, Cunha \& Stedefeltd, 2013; Ministério da Saúde, 2004; Ministério da Saúde, 2006; Pistore \& Gelinskib, 2006). Entretanto, questiona-se: as ações realizadas com o objetivo de aprimorar e promover a execução do PNAE na esfera normativa foram acompanhadas pela adequação dos aspectos operacionais?

Partindo dessa questão, este artigo tem como objetivo analisar o processo de transição pelo qual passou a alimentação escolar ao longo dos anos, considerando as modificações ocorridas na estrutura do seu espaço de produção.

\section{Metodologia}

Trata-se de um ensaio oriundo de uma investigação empírica, com abordagem socioantropológica. A pesquisa de campo, de cunho etnográfico, foi desenvolvida no período de outubro a dezembro de 2014, em uma escola pública localizada em bairro popular da cidade de Salvador (BA).

Participaram do estudo as três merendeiras que trabalhavam no local, com idades de 34,45 e 55 anos, com Ensino Médio completo e desempenhavam tal função há um período entre 12 e 20 anos. Os dados foram registrados em diário de campo e gravador digital, cujo áudio foi transcrito e sistematizado para análise. Além disso, foram analisadas publicações científicas e documentos oficiais para estabelecer uma interlocução teórica com os dados produzidos em campo (observação participante e entrevistas).

A pesquisa foi aprovada pelo Comitê de Ética em Pesquisa da Escola de Nutrição (parecer 730.150) e conduzido de acordo com a Resolução no 466 do Ministério da Saúde (2012). A instituição de ensino não foi revelada e os nomes das interlocutoras foram substituídos por pseudônimos. 


\section{Resultados e Discussão}

\subsection{Da "cozinha ideal" à "cozinha real": o espaço de trabalho entre a norma e a estrutura.}

A alteração na denominação de "cantina" para "Unidade de Alimentação e Nutrição" (UAN), subsidiada por marcos legais, revela uma mudança de titulação na tentativa de conferir um caráter mais profissional e científico assentado nas bases das Ciências da Nutrição. Contudo, as ações realizadas no sentido de aprimorar e promover a execução do PNAE, que ocorreram, em grande parte, no âmbito normativo, não foram acompanhadas pela adequação dos aspectos operacionais. Tal disparidade evidencia que a transição ainda não se concretizou efetivamente (Cardoso, Souza \& Santos, 2005; Colombo, Oliveira \& Silva, 2009; Da Cunha, Stedefeldt \& De Rosso, 2012; Danelon, Danelon \& Silva, 2006; Lima, Cunha \& Stedefeltd, 2013; Ministério da Saúde, 2004; Ministério da Saúde, 2006; Pistore \& Gelinskib, 2006).

Ainda assim, conforme Lima, Cunha e Stedefeltd (2013), no Brasil, ainda não há uma legislação específica. A UAN, produtora de refeições para creches ou escolas, atende aos requisitos da RDC n 216/ANVISA (Ministério da Saúde, 2004), criada com o objetivo de "estabelecer procedimentos de Boas Práticas para serviços de alimentação, a fim de garantir as condições higiênico-sanitárias do alimento preparado" (Ministério da Saúde, 2004). No entanto, o vasto âmbito de aplicação dessa legislação equipara as "cantinas" a outros setores da alimentação coletiva com diferentes graus de complexidade: "cozinhas industriais, cozinhas institucionais [...] pastelarias, restaurantes, rotisserias e congêneres" e exige a execução de regras de modo unânime.

Adicionalmente, Da Cunha, Stedefeltd e De Rosso (2012) identificaram a "falta de um instrumento-padrão para a avaliação das Boas Práticas na alimentação escolar" e ressaltaram que isso "pode dificultar o trabalho do nutricionista", que precisa identificar o grau de cumprimentos das normas. Nesse sentido, os Centros Colaboradores em Alimentação e Nutrição do Escolar, do Rio Grande Sul (CECANE UFRGS) e São Paulo (CECANE UNIFESP), elaboraram o "Guia de Instruções das Ferramentas para as Boas Práticas na Alimentação Escolar", com o objetivo de subsidiar o "fornecimento de uma alimentação escolar segura, do ponto de vista higiênico-sanitário" (CECANE UFRGS \& CECANE UNIFESP, 2013). Entretanto, diante das distintas realidades que compõem o cenário do PNAE no território nacional, tal dispositivo não se mostrou capaz de atender às especificidades e características inerentes a esse tipo de serviço.

Neste contexto, segundo publicações científicas (Lima, Cunha \& Stedefeltd, 2013; Sousa, Amóra \& Siqueira, 2012), as merendeiras ainda são consideradas as maiores responsáveis pela contaminação dos alimentos, podendo ocasionar surtos de doenças de origem alimentar. Lima, Cunha e Stedefeltd (2013) verificaram que a principal inadequação encontrada nos estabelecimentos referente à higienização das mãos era a não utilização de materiais específicos para o procedimento "por falta ou do produto ou de informação por parte do manipulador quanto ao uso correto dos mesmos". Desse modo, apesar de sinalizarem os problemas vivenciados nas cozinhas escolares, são recorrentes o discurso de culpabilização destes profissionais e a defesa da realização de treinamentos contínuos e sistemáticos para um melhor desempenho de suas atividades.

Com base na legislação sanitária, são elaborados treinamentos com o objetivo de instrumentalizar esses sujeitos a respeito das normas que constituem as Boas Práticas de Manipulação de Alimentos (Ferro, Fialho, Pires, Teles \& Santos, 2018; Oliveira et al., 2019). Gabriel, Santos, Vasconcelos, Milanez e Hulse (2010) enfatizaram que esses profissionais "precisam ser capacitados e preparados para trabalhar com alimentação escolar, de modo que essas ações possibilitem uma conscientização e entendimento por parte deles" acerca da manipulação adequada dos alimentos. No entanto, a despeito das condições concretas de trabalho, como destacaram Lima, Cunha e Stedefeltd (2013), esses estabelecimentos, em sua maioria, assemelham-se "mais às cozinhas domésticas do que às unidades industriais", o que torna ainda mais difícil o cumprimento das exigências da legislação vigente.

Em relação ao local do presente estudo, não foram encontrados dados históricos, mas, conforme relato das merendeiras e informações do endereço eletrônico da instituição, o prédio da escola, que atualmente atende 800 estudantes, 
segundo informação da direção da escola, foi edificado ainda na década de 1950, quando o bairro estava em franco crescimento, estimulado por reformas urbanas. Quarenta anos depois, as instalações foram apropriadas pela Prefeitura do município em estudo, que, desde então, sob diversas gestões, tem realizado pequenas reformas consideradas insuficientes para o seu pleno funcionamento.

Alagamentos são periodicamente referidos pelos noticiários locais como uma das sérias consequências das falhas estruturais, em que há áreas desativadas ou impróprias ao desenvolvimento das atividades. Desse modo, o espaço destinado à produção de alimentos foi construído há mais de meio século ainda como "cantina", não apenas no sentido primordial da concepção do termo, mas também em relação à edificação estrutural.

A observação em campo permitiu identificar que, apesar de existir uma placa afixada na porta de entrada com a inscrição "cantina", as merendeiras, estudantes, gestores e funcionários da escola referiam-se ao local como "cozinha". É possível que essa mudança da denominação esteja associada à maior utilização e processamento de alimentos in natura em comparação ao uso de produtos industrializados. Soma-se a isso que, embora o aumento da demanda da produção de refeições tenha contribuído para o incremento do volume de trabalho, o espaço edificado há quase 65 anos não sofreu significativas modificações estruturais.

O comprometimento do sistema hidráulico pode representar alguns dos problemas da realidade local. A pia usada para higienização de alimentos e utensílios apresentava danos na base de granito que servia como apoio e impossibilitava a fixação de uma das cubas, cuja sustentação era mantida por um cabo de vassoura cortado ao meio, amparado pelo piso. Um balde colocado sob a pia fazia a contenção da água proveniente do constante vazamento do sifão. A outra cuba apresentava problemas na vedação da torneira, mantida amarrada por um pedaço de plástico que, apesar de controlar o intermitente fluxo de água, dificultava o seu uso.

Avarias no revestimento externo, mobiliário e equipamentos em precário estado de conservação e a insuficiência de materiais e utensílios expõem adicionais deficiências de diferentes ordens, que impõem estratégias de improvisação e adaptação no cotidiano de trabalho das merendeiras.

Diante disso, para as interlocutoras deste estudo, as tentativas de adoção de atitudes e práticas higiênicas, de acordo com as normas, eram justificadas pela preocupação quanto às possíveis sanções decorrentes da verificação pela nutricionista e pela "saúde pública", modo como faziam alusão ao fiscal da Vigilância Sanitária. O discurso de culpabilização influencia as ações de vigilância das suas atividades, o que tem gerado práticas permeadas por conflitos e pelo receio quanto às possíveis sanções decorrentes do que, embora esteja situado no campo do possível, pode ser considerado transgressão, diante de avaliações restritas e dicotômicas.

Esse fato evidencia a permanência do estigma da ação policialesca exercida pelos órgãos de fiscalização, reminiscência do final do século XIX, quando, segundo Rodrigues (1995, p.47), o governo estabelecia uma espécie de policiamento para a vigilância da limpeza urbana, que, por sua vez, identificava e castigava "os que não se deixaram convencer". Cabe refletir sobre o paradoxal papel do Estado neste contexto: O mesmo Estado que não provem melhorias na estrutura física, provisionamento adequado de equipamentos e utensílios de forma a oferecer as condições para a produção da alimentação de forma segura, é o mesmo que elabora as normas, fiscaliza e pune os estabelecimentos produtores de alimentação, neste caso, a UAN/cozinha/cantina escolar.

As instruções quanto às regras a serem seguidas, para essas interlocutoras, têm gerado a expectativa de "um dia" poderem trabalhar em uma "cozinha ideal". Na visão das mesmas, somente após a sua "reconstrução", esse espaço atenderia plenamente aos requisitos normatizados pela setorização física, com especificidades de segregação de materiais, objetos e atividades. Seria esse o local para o desenvolvimento integral de práticas reforçadas nos treinamentos dos quais participavam periodicamente, conforme a lógica que fundamentava as ações educativas. As disparidades entre a "cozinha ideal" e a "cozinha 
real" evidenciam o distanciamento entre a teoria (legislação e conteúdo das capacitações) e a prática (realidade vivenciada no cotidiano).

Neste contexto, é importante ressaltar que a legislação e o discurso científico são produtos culturais com raízes históricas, assim como os princípios que norteiam as práticas desenvolvidas pelas merendeiras ao elaborarem estratégias para tentar aproximar a "cozinha real" da "cozinha ideal", explicitando um modo particular de lidar com as normas da UAN sendo ainda "cantina".

\section{Considerações Finais}

Em maior proporção do que as campanhas e programas que o antecederam, o PNAE passou por transformações importantes. A revisão e atualização de parâmetros nutricionais, o aumento significativo do volume e a complexidade das refeições demandaram ações no sentido de aprimorar e cumprir os objetivos do Programa. Além disso, houve uma disparidade das alterações realizadas no âmbito normativo e modificações concretizadas nos aspectos operacionais. Como consequência, a transição de "cantina" para "Unidade de Alimentação e Nutrição" prevaleceu muito mais no campo conceitual do que no estrutural. Com isso, ocasiona o surgimento de dois espaços simbólicos: a "cozinha ideal”, plenamente alinhada à legislação e ao conteúdo das capacitações, e a "cozinha real", em que são realizadas as práticas cotidianas.

A aproximação etnográfica associada à pesquisa documental permitiu identificar que, ainda que tenha o objetivo de garantir a alimentação escolar como direito dos estudantes, a mesma é vista de forma fragmentada. Assim, a produção da "merenda" se modifica ao longo dos anos e nem sempre está relacionada com o contexto socioeconômico e cultural, que também interfere na realidade escolar, produzindo distanciamento entre o que o Programa se propõe e as condições em que tem sido executado.

O descompasso entre os avanços nas dimensões conceituais e estruturais do PNAE revela o distanciamento entre a legislação e a realidade. Por conseguinte, o fato de uma transição não ter ocorrido efetivamente no campo estrutural tem gerado a criação de um espaço quimérico no imaginário das merendeiras, demasiado discrepante do local de trabalho ainda marcado pela precariedade. A constância de múltiplas contingências provoca improvisos e adaptações no cotidiano de trabalho, originando "microfissuras" nos limites estabelecidos pela norma institucional, e demonstra a sua insuficiente compatibilidade com o contexto socioeconômico e cultural que ainda afeta a realidade escolar.

Este estudo analisou o processo de transição pelo qual passou a alimentação escolar ao longo dos anos, considerando as modificações ocorridas na estrutura do seu espaço de produção a partir da observação participante e entrevistas com merendeiras de uma escola pública. Como limitações desta investigação, embora a pesquisa evidencie aspectos relevantes desta realidade, é importante reconhecer que se trata de um recorte. Ademais, a revisão teórica realizada ao longo deste trabalho, revelou a escassez de pesquisas qualitativas sobre o objeto estudado, o que restringiu a interlocução teórica. Nesse sentido, sugere-se investigações neste campo e que avanços posteriores na política e gestão da alimentação escolar devem considerar a escuta de todos envolvidos no processo, além de uma reflexão crítica sobre as transições propostas, a respeito dos seus objetivos e efetividade no âmbito escolar.

\section{Referências}

Cardoso, R. de C. V., Souza, E. V. A. de \& Santos, P. Q. dos. (2005). Unidades de alimentação e nutrição nos campi da Universidade Federal da Bahia: um estudo sob a perspectiva do alimento seguro. Revista de Nutrição, 18 (5), 669-680.

Centro Colaborador em Alimentação e Nutrição do Escolar da Universidade Federal do Rio Grande do Sul \& Centro Colaborador em Alimentação e Nutrição do Escolar da Universidade Federal de São Paulo. (2013). Guia de Instruçães das Ferramentas para as Boas Práticas na Alimentação Escolar. http://www.fnde.gov.br/programas/alimentacao-escolar/alimentacao-escolar-material-de-divulgacao/alimentacao-manuais/item/5320-ferramenta-de-boaspráticas-de-fabricação-de-alimento 
Chaves, F. M. (2000). Outros olhares em escolas públicas: as relações sociais de trabalho sob a ótica de merendeiras e serventes. Trabalho \& Educação, 7(7), $132-156$.

Coimbra, M., Meira, J. F. P. de, \& Starling, M. B. de L. (1982). Comer e aprender: uma história da alimentação escolar no Brasil. Belo Horizonte, MG: INAE.

Colombo, M., Oliveira, K. M. P. de \& Silva, D. L. D. da. (2009). Conhecimento das merendeiras de Santa Fé, PR, sobre higiene e boas práticas de fabricação na produção de alimentos. Revista Higiene Alimentar, 23 (170/171), 39-46.

Costa, E. de Q. (2001). A dimensão educativa do trabalho das merendeiras: análise do material instrucional elaborado pelo Instituto de Nutrição Annes Dias. Dissertação de mestrado, Universidade Federal do Rio de Janeiro.

Da Cunha, D. T., Stedefeldt, E. \& De Rosso, V. V. (2012). Boas práticas e qualidade microbiológica nos serviços de alimentação escolar: uma revisão sistemática. Revista Brasileira de Pesquisa em Saúde, 14 (4), 108-121.

Danelon, M. A. S., Danelon, M. S. \& Silva, M. V. (2006). Serviços de alimentação destinados ao público escolar: análise da convivência do Programa de Alimentação Escolar e das cantinas. Segurança alimentar e nutricional, 13 (1), 85-94.

Fernandes, A. G. de S. (2012). Alimentando o saber: o perfil das merendeiras, suas percepções em relação ao ofício e as possibilidades para a educação. Dissertação de mestrado, Universidade Federal do Rio de Janeiro.

Ferro, L. L., Fialho, C. J., Pires, C. R. F., Teles, N. B., \& Santos, V. F. (2018). Condições higiênico sanitárias de Unidades de Alimentação e Nutrição de escolas públicas do estado do Tocantins. Segurança alimentar e nutricional, 5 (2),118-130.

Gabriel, C. G., Vasconcelos, F. A.G. de, Milanez, G. H. G. \& Hulse, S. B. (2010). Cantinas escolares de Florianópolis: existência e produtos comercializados após a instituição da lei de regulamentação. Revista de Nutrição, 23 (2), 191-199.

Gomes, K. dos S. (2014). Cozinhando e dialogando: merendeiras, suas possibilidades e desafios para ações de educação alimentar e nutricional em escolas públicas do Rio de Janeiro. Dissertação de mestrado, Universidade Federal do Rio de Janeiro.

Lei n. 11.947, de 16 de junho de 2009. (2009). Dispõe sobre o atendimento da alimentação escolar e do Programa Dinheiro Direto na Escola aos alunos da educação básica; altera as Leis $\mathrm{n}^{\circ}$ 10.880, de 9 de junho de 2004, 11.273, de 6 de fevereiro de 2006, 11.507, de 20 de julho de 2007; revoga dispositivos da Medida Provisória nº 2.178-36, de 24 de agosto de 2001, e a Lei no 8.913, de 12 de julho de 1994; e dá outras providências.

Lima, A. F. A. de, Cunha, D. T. da \& Stedefeldt, E. (2013). Avaliação do risco sanitário em Unidades de Alimentação e Nutrição Escolar da Baixada Santista, SP. Revista Higiene Alimentar, 27 (218/219), 51-56.

Ministério da Saúde. (2004). Resolução RDC $n^{\circ}$ 216, de 15 de setembro de 2004. Dispõe sobre Regulamento Técnico de Boas Práticas para Serviços de Alimentação. Agência Nacional de Vigilância Sanitária.

Ministério da Saúde. (2006). Portaria Interministerial $n^{\circ} 1.010$, de 8 de maio de 2006. Institui as diretrizes para a promoção da alimentação saudável nas escolas de educação infantil, fundamental e nível médio das redes públicas e privadas, em âmbito nacional. http://dtr2001.saude.gov.br/sas/PORTARIAS/Port2006/GM/GM-1010.htm

Ministério da Saúde. (2012) Resolução $n^{\circ} 466$, de 12 de dezembro de 2012. Aprovar as diretrizes e normas regulamentadoras de pesquisas envolvendo seres humanos. Conselho Nacional da Saúde. Brasília, DF.

Moysés, M. A., \& Collares, C. (1997). Desnutrição, fracasso escolar e merenda. In: Patto, M. H. (Org.) Introdução à psicologia escolar (2a ed.). Casa do Psicólogo.

Oliveira, A. S. S. S., Macedo, J. L., Pereira, I. C., Soares, E. L. P., Gomes, F. O., \& Assunção, M. J. S. M. (2019). Research, Society and Development, 8(3). https://rsdjournal.org/index.php/rsd/article/view/830. doi: http://dx.doi.org/10.33448/rsd-v8i3.830

Pistore, A.R. \& Gelinskib, J.M.L.N. (2006). Avaliação dos conhecimentos higiênico-sanitários de manipuladores de merenda escolar: fundamento para treinamento contínuo e adequado. Revista Higiene Alimentar, 20 (146), 17-20.

Rodrigues, J. C. (1995). Higiene e ilusão: O lixo como invento social. Nau.

Sousa, A. M. F., Amóra, S. S. A. \& Siqueira, E. E. (2012). Percepção das merendeiras sobre boas práticas de manipulação de alimentos em unidades de educação infantil. Anais do I Congresso Internacional Interdisciplinar em Sociais e Humanidades, 1, 1-20. 